\title{
Current Situation of Rhythmic Gymnastics Teaching in Colleges and Its Measures
}

\author{
Liying Shen \\ Changzhou Campus \\ Hohai University \\ Changzhou, China 213022
}

\begin{abstract}
The Rhythmic Gymnastics course opened for physical education colleges is beneficial to cultivation of students' external temperament and their overall development, which is suitable for students' mental health development demands; however, the current teaching of college Rhythmic Gymnastics is facing a great dilemma, thus how to adapt to new forms of reform and development has become an important topic of college physical education; some solutions and means have been proposed in this paper from review of rhythmic gymnastics development and analysis of college rhythmic gymnastics course, with appropriate recommendations and strategies being provided for teaching of college rhythmic gymnastics.
\end{abstract}

Keywords - college physical education; Rhythmic Gymnastics; teaching; current situation; measures

\section{INTRODUCTION}

College students will be the main backbone in various industries in our country after graduation, and various colleges shall attach great importance to physical education for regular college students according to their own actual conditions. Make physical education a life-long process for college students, promote the course system of college physical education more perfect and cultivate senior talents who adapt to the need of new economic society and socialist modernization construction. Rhythmic gymnastics emphasizes beautiful movements as well as correct and straight body posture, which can be divided into the mass rhythmic gymnastics and athletics rhythmic gymnastics on the basis of natural movements of the body and selfexpression. Among them, the mass rhythmic gymnastics is also known as general rhythmic gymnastics, which highlights the fitness effect with strong character of times. Therefore, it is a noble and elegant sports fitness program with its unique charm, namely, to integrate the fitness, entertainment, and performance into a unity with various forms and rich contents, so it is a sport suitable for the vast number of teenagers as well as middle-aged and elderly. Therefore, opening rhythmic gymnastics course in colleges is a positive means to adapt to the times and to cultivate students' comprehensive ability. In the tide of teaching reform, each teacher of rhythmic gymnastics in colleges shall strive to improve their professional accomplishment, make this new-rising subject more perfect and make it an important part of mass entertainment fitness. However, in many Chinese colleges, rhythmic gymnastics haven't been opened and taught very well. Therefore, how to reform the teaching of rhythmic gymnastics, let the students know more about rhythmic gymnastics, and better cultivate students' interest in rhythmic gymnastics, so as to promote the benign development of the mass rhythmic gymnastics, make contribute to students' lifelong sports, is a problem worthy of studying.

\section{INTRODUCTION OF RHYTHMIC GYMNASTICS DEVELOPMENT}

Rhythmic gymnastics hasn't been developed for long as an independent women's sport, but it already has a history of over 100 years as an integral part of women's gymnastics and a means for physical education with constant high attention from many countries: Currently, many countries have listed rhythmic gymnastics into teaching program for physical education of schools at various levels, making it a means for women's physical education by regulation. Especially the former Soviet Union and Bulgaria have not only listed rhythmic gymnastics into the teaching program for physical education of colleges, primary and secondary schools in the 1930s, but also offer exercise of rhythmic gymnastics in kindergarten - taking will and character exercising and aesthetic awareness cultivation as basic courses. It is more popular in Bulgaria, as 1 per 900 person is engaged in rhythmic gymnastics. In previous major international rhythmic gymnastics competition, almost all the high-level athletes come from Bulgaria and Russia. The mass rhythmic gymnastics performances with participants of different age groups can often be seen in Netherlands, Denmark, Sweden and other European countries. The men's rhythmic gymnastics class has been opened in Japan Oriental Sports University and Department of Physical Education of the University of Tsukuba, making the 125 rhythmic gymnastics clubs in Japan have a male member. Due to men's participation in rhythmic gymnastics, the characteristic of simple feminine of rhythmic gymnastics has been changed, adding masculinity in the grace of rhythmic gymnastics. Obviously, many countries have attached great importance to rhythmic gymnastics, and China is no exception. After the rhythmic gymnastics was introduced in China in early 1950s from the former Soviet Union, the rhythmic gymnastics courses have been opened in colleges, primary and secondary schools one after another, becoming the teaching 
contents of physical education of schools at all levels with the attention it deserves. Especially in the early 1980s to the mid-1990s, rhythmic gymnastics is very popular in China's colleges, primary and secondary schools.

\section{Dilemma OF COLLEGE RHYTHMiC GyMnASTICS TEACHING}

Rhythmic gymnastics has played an important role in physical education contents in Chinese colleges for long. But in the actual conduct process, it is not optimistic. With the emerging of new sporting events and the acceptance by school physical education, making rhythmic gymnastics in school being ignored gradually, and some even unable to carry it out. The requirement of mass rhythmic gymnastics participants' physical fitness and athletic ability is low. While enhancing people's physical and psychological health and improving their physical fitness, it can also cultivate people's artistic expression and elegant manner as well, meeting woman's physical and mental characteristics very well, which is very suitable for female college students as a training tool. However, most regular college students come from ordinary high schools, and they have a poor sports foundation, general physical fitness, and lower levels of physical fitness, physical function and athletic ability, coupled with the rhythmic gymnastics is different from such popular sports in students as ball games and track and field, it is even a previously unknown concept, thus rendering the students a slow acceptance and comprehension of teaching methods in learning rhythmic gymnastics with more difficulties, resulting in such situations as low enthusiasm, lack of self-confidence, poor motivation of students in class, thus the classroom teaching effect is affected to some degree.

Reviewing the research history of rhythmic gymnastics, relevant theory and practice research of teaching effect about rhythmic gymnastics have achieved a certain degree of progress, learning from these relevant studies will be of great referential meaning for this paper. But for now, there are still many problems, especially studies on teaching effects of optional course of college popular rhythmic gymnastic is still very weak, which is obviously not conducive to the development of regular course of college rhythmic gymnastic. Coupled with the teaching of rhythmic gymnastic has followed the training mode of athletics rhythmic gymnastics all along, and along with the development of times, the demand of physical education by society has experienced a huge change, and people have changed their concept about fitness, thus the requirements for selecting their favorite fitness programs have become increasingly high. Yet the rhythmic gymnastics course in colleges has failed to make timely adjustments and updating, making many rhythmic gymnastics teaching content break away from the actual social needs. Contradictions have been generated between rhythmic gymnastics teaching and social needs, and people have a growing demand for entertainment and fitness gymnastics, while rhythmic gymnastics teaching is the same as other sports teaching with sport technique as the center. Through research, it can be found that there are many factors restricting the teaching effect, such as teachers, students, curriculum setting, environmental impact and other factors. The interact degree and relationship among these factors remain to be studied further in-depth. In short, only appropriate adjustments targeting the teaching effect of optional course of rhythmic gymnastics is made, and improvement and enhancement from various aspects is made one by one, can the teaching effect of general course of rhythmic gymnastics be improved effectively.

\section{REFORM OF COLLEGE RHYTHMIC GYMNASTICS AND MEASURES}

It can be learned through investigation of the teaching program and teachers' teaching plan of various college rhythmic gymnastics and other related information that, the teaching objectives positioning of rhythmic gymnastics courses in many colleges is being influenced by traditional thinking habits of paying attention to skills and achieving level requirements, focusing on technological learning and short-term benefits, neglecting breadth and extensibility of various basic movements learning, lack of understanding about social demand and positioning of teaching focus, ignoring cultivation of practical skills. Although students have learned a lot of movements, but when encountered some problems about popularization and promotion, they will be unable to adapt. Under today's social background of neglecting athletics and focusing on fitness by colleges, primary and secondary schools as well as constant enhancement of social group's fitness consciousness, current college rhythmic gymnastics teaching in China is still remain in the teaching movements themselves, making current rhythmic gymnastics teaching unable to fully play its due role. Meanwhile, as a unique sports culture, rhythmic gymnastics takes the natural and graceful body movements as foundation with unique aesthetic and entertainment role. Current positioning of teaching objectives of college rhythmic gymnastics has failed to reflect the connotation of humanistic spirit, which need to reexamine this issue in terms of teaching objectives.

\section{A. Strengthen Training of Teachers to Improve the Level of Rhythmic Gymnastics Teachers}

Rhythmic gymnastics teachers play a vital role in the conduct of rhythmic gymnastics. The level of rhythmic gymnastics teachers is directly related to the level of scientific training of rhythmic gymnastics and the improvement of athletic level. Currently, the teaching materials are all selected by the teachers themselves as there is no uniform teaching materials and teaching program of rhythmic gymnastics in our province. Teachers' work is creative, with the development of sports, the teaching, training theory and teaching, training practice is also moving forward constantly, and the teachers with poor professional ability, out-of-date information, unscientific teaching and training methods, rare participation in training will be eliminated by the society. And all the abovementioned are the obvious deficiencies existing in Chinese rhythmic gymnastics teachers. 


\section{B. Change the Teaching Organizational Form to Establish a Good Teaching Order}

Teaching organization of rhythmic gymnastics shall also follow the general teaching law and teaching principles, namely to always conduct it around students' learning interests and rhythmic gymnastics features in terms of form. Rhythmic gymnastics itself has versatility, therefore, integrated teaching shall be adopted as appropriate, and the so-called "integrated education" is to teach the variety of tasks and contents in integration. Integrated teaching content tends to change a lot, and is easy to stimulate students' interest in learning with more close teaching organization. Therefore, it shall be conducted step by step with emphasis and well arranged level in a simple but profound manner. Especially at the time of routine learning, it must be conducted in reasonable order. Students shall be organized to experience movements following the teacher's rational explanation and demonstration, seeking the feeling of perfect and harmony. In the learning process of movement rhythm, students shall be required to learn shouting beat of various movements first, but also be required to understand different beat of various music. And then they shall be taught on basis of their correct mastering of movements. Render varied rhythmic to the basic rhythm of movements, making it possessing its own characteristics with more charm and comparison changes of the pace, strength, and size of the stride movements etc..

\section{Application of New Teaching Means and Methods}

First of all, rhythmic gymnastics has an attribute of physical education, and many effective teaching methods in physical education still apply to rhythmic gymnastics. And rhythmic gymnastics is a movement with dance as its main content, therefore, it also has entertainment properties, namely rhythm, normative and entertaining. Thus it has a distinctive dual features in terms of selection and application of teaching methods. Therefore, teaching methods and means of rhythmic gymnastics shall be different from that of other projects. Rhythmic gymnastics is an elegant and civilized exercise for bodybuilding, and the demonstration capacity of teachers and music quality will directly affect the students' interest in learning and learning effect. And that has determined the teaching of rhythmic gymnastics shall not be a simple explanation, demonstration and practice, yet it shall be taught with integration of a variety of teaching methods based on the actual situation of teaching, focusing on coaching and guiding, while paying attention to the combination of basic movements with music. (1) Individualized education, easy things come first: Since rhythmic gymnastics is bare-handed or with equipment involving a variety of contents, therefore, the teaching must be conducted based on specific circumstances of the students, such as gender, physical quality, technical level and type of education and culture to appropriately and reasonably select teaching materials, and to teach with different methods. (2) Pay attention to teachers' performance and guiding role: Emphasis shall be laid on teachers' performance and leading role, which plays an important role for students to get an intuitive feeling about rhythmic gymnastics, such as emotional and full of spirit, solemn and form-fitting clothes, graceful dance demonstration, and accurate beat command, accurate movement teaching and timely error correction, etc., which plays a very important role in stimulating students' interest in learning, promoting them to love rhythmic gymnastics more and the successful completion of teaching task. (3) Pay attention to the cultivation of students' ability: Take full advantage of questioning, observation, evaluation, interaction and guidance arrangement and others, to stimulate and inspire students timely after students have mastered certain skills, make students participate in simple choreography exercises, which is helpful to stimulate students' enthusiasm in learning and their creativity.(4) Strengthen the application of modern teaching methods in rhythmic gymnastics: Appropriate electronic teaching content shall be arranged in the teaching process. On the one hand, to further stimulate and mobilize the students' interest in learning by watching a high level and large scale competition; and on the other, technical difficulties can be resolved through audio-visual media, to enable students to learn the internal structure of technical movements, movement track and methods more comprehensively and visually, to make technical movements be mastered more easier, more accurate and more efficient.

\section{Adjust Course Structure Using Diversified Curriculum Evaluation}

To achieve healthy physical education curriculum objectives, the extracurricular physical exercise shall be integrated with rhythmic gymnastics courses with purpose, plan and organization, and involve the sports training, small and diverse activities into the rhythmic gymnastics course, to form the course structure organically link the internal and external courses both inside and outside school. Diversified teaching evaluation system. Simple evaluation system will inevitably lead to poor learning content, methods and means, resulting in students' thin knowledge structure. Therefore, current reform of "result type" evaluation shall be the integration of "results" testing and "process" testing. Examination of rhythmic gymnastics shall not only focus on passing the movement levels, yet it shall combine the written examination of theoretical knowledge, methods application methods, technical grade standards, teaching practice ability survey and their own choreography, to establish a diversified teaching evaluation system to adapt to future requirements of social market in terms of teaching.

\section{CONCLUSION}

In teaching college rhythmic gymnastics, we shall focus on cultivation of students' understanding of the rhythmic gymnastics project itself, establish a correct motivation to learn, gradually develop their interest in learning rhythmic gymnastics, thus to realize active learning. Rhythmic gymnastics teachers shall constantly improve themselves, enrich their expertise, and they shall be innovative to continuously change their teaching concepts, methods and means. And they shall teach students the learning methods to guide students to learn actively, while constantly enriching their knowledge and internal quality to increase their charm of personality. Teachers shall keep pace with the times and 
select teaching materials that are interesting with epochal character, performing and popularity. And they shall improve allocation of hours, add theory class hours as appropriate and perfect evaluation system using a wide range of evaluation methods, making teaching evaluation reflect true level of the students as much as possible.

\section{REFERENCES}

[1] Zhang Qunhua. Discussion on Key Points for Improving Teaching Effect of Rhythmic Gymnastics [J]. Sport Science and Technology, 2006 (12)

[2] Zhu Xiaoyong. Study on Scientificity of Teaching [J]. Southwest University, 2011(3)

[3] Shi Mingya. Improvement of Rhythmic Gymnastics Teaching [J]. Journal of Sports and Science, 2002(6).

[4] Xu Xiudi. Survey and Analysis on Teaching Effect of College Rhythmic Gymnastics[J]. Journal of Shanghai University of Sport, 2005(3) 